\title{
Reducing medication errors in critical care: a multimodal approach
}

REVIEW

\section{Rachel M Kruer' \\ Andrew S Jarrell' \\ Asad Latif 2,3}

'Department of Pharmacy, The Johns Hopkins Hospital, Baltimore, MD,

USA; '2Department of Anesthesiology and Critical Care Medicine, Johns Hopkins University School of Medicine, Baltimore, MD, USA; ${ }^{3}$ Armstrong Institute for Patient Safety and Quality, Johns Hopkins Medicine, Baltimore, MD, USA

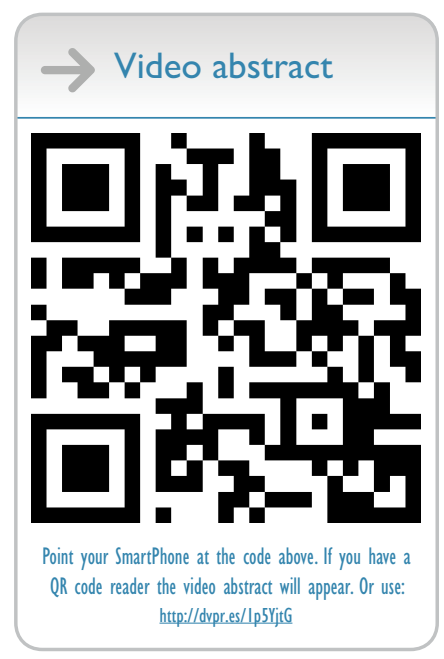

Correspondence: Asad Latif Department of Anesthesiology and Critical Care Medicine, Johns Hopkins University School of Medicine,

600 North Wolfe Street, Meyer 297-A,

Baltimore, MD 21287, USA

Tel +l 4105022714

Fax + I 4109558978

Email alatifl@jhmi.edu
This article was published in the following Dove Press journal:

Clinical Pharmacology: Advances and Applications

I September 2014

Number of times this article has been viewed
Abstract: The Institute of Medicine has reported that medication errors are the single most common type of error in health care, representing $19 \%$ of all adverse events, while accounting for over 7,000 deaths annually. The frequency of medication errors in adult intensive care units can be as high as 947 per 1,000 patient-days, with a median of 105.9 per 1,000 patient-days. The formulation of drugs is a potential contributor to medication errors. Challenges related to drug formulation are specific to the various routes of medication administration, though errors associated with medication appearance and labeling occur among all drug formulations and routes of administration. Addressing these multifaceted challenges requires a multimodal approach. Changes in technology, training, systems, and safety culture are all strategies to potentially reduce medication errors related to drug formulation in the intensive care unit.

Keywords: medication safety, drug design, drug formulation, patient safety

\section{Introduction}

At the beginning of the current millennium, the Institute of Medicine (IOM) started a series of reports on quality in health care. The issue of medication safety was highlighted by the first report, To Err is Human: Building a Safer Health System. ${ }^{1}$ It was estimated that unintentional errors caused as many as 44,000 to 98,000 deaths per year, making them at least the 8 th leading cause of death in the US. ${ }^{2}$ Medication errors were identified as the single most common type of error in health care, representing $19 \%$ of all adverse events and accounting for more than 7,000 deaths annually. ${ }^{3-5}$ Subsequent reviews have conservatively estimated that 1.5 million preventable adverse drug events (ADEs) occur each year in the US, with hospitalized patients suffering at least 400,000 ADEs per year. This figure equates to one medication error per patient per day. ${ }^{6}$ Although not all ADEs cause harm, those that do can be costly in terms of direct, indirect, and opportunity costs. Hospital costs alone for preventable ADEs in admitted patients are approximately $\$ 3.5$ billion across the country. ${ }^{6}$ In ambulatory settings, the annual cost of drug-related morbidity and mortality was estimated to be $\$ 177.4$ billion in 2000 , and rising. ${ }^{7}$

In its sentinel report, the IOM raised the issue of product design. It recommended that the US Food and Drug Administration (FDA) develop and enforce standards for drug packaging and labeling that would optimize their safe use, and that pharmaceutical companies be required to formally test proposed names to avoid confusion. ${ }^{1}$ These recommendations were echoed by the Secretary of Health and Human Services, who asked that all FDA-regulated products adopt safe labeling practices to decrease the risk of ADEs and improve patient safety. ${ }^{8}$ The enormous magnitude of the problem led 
Congress to mandate the Center for Medicare and Medicaid Services to sponsor a comprehensive study of drug safety and quality issues by the IOM in 2004. The resultant 2007 report, Preventing Medication Errors, comprehensively addressed the actions needed to achieve the next level in medication safety. ${ }^{6}$ The IOM found that $33 \%$ of medication errors and $30 \%$ of resultant fatalities were secondary to labeling and packaging issues. ${ }^{6}$ Along with system, process, and reporting changes, their recommendations included product-specific measures designed for the end user, such as incorporating cognitive and human factors engineering principles to address issues with presentation of information in labeling and nomenclature.

\section{Challenges encountered in the intensive care unit}

Critical care areas present a particular challenge with regard to medication errors. ${ }^{9}$ They are a dynamic environment with critically ill patients who often require rapid adaptation of on-going management. Intensive care units (ICUs) can be error-prone settings, where even otherwise minor adverse events can lead to serious disability. ${ }^{4,10,11}$ The frequency of medication errors in adult ICUs can be as high as 947 per 1,000 patient-days, with a median of 105.9 per 1,000 patientdays. ${ }^{12-14}$ Medication errors are estimated to account for $78 \%$ of all medical errors in ICUs, with an average of 1.75 medication errors per patient per day. ${ }^{15}$ Not only are medication errors more frequent in ICU settings than in non-ICU settings, they are also more likely to be severe and cause harm. ${ }^{16}$

Many factors are hypothesized to contribute to the relatively high incidence and associated morbidity of medication errors in the ICU. The patients themselves are the most complex and critically ill in the hospital setting. ${ }^{15}$ By virtue of being sicker, older, and having more comorbidities, these patients are less resilient to errors. Because they require a higher intensity of care provision and may receive more medications, they may be at greater risk of iatrogenic harm. ${ }^{11}$ Pharmacokinetics of medications can also be altered in critically ill patients, principally through changes in volume of distribution and drug clearance. ${ }^{17}$ Large volume resuscitations, positive pressure ventilation, surgical procedures, systemic inflammatory response, and changes in protein binding, all common in ICU patients, affect the pharmacokinetics of many drugs. ${ }^{18}$ In addition, these patients are usually unable to help facilitate their own care, a problem aggravated by the volume of transfers to and from ICUs. ${ }^{19-21}$ Medication safety in ICUs might also be compromised because of the risks associated with the use of multiple medications per patient and the use of high-risk drugs associated with potentially severe adverse events. Drugs used in the ICU are more likely to be potent, require dose calculations, have medication interactions, and be continuous infusions (which have a greater potential for error). ${ }^{22}$ Many medications may be used for off-label indications in the ICU setting, similar to the non-ICU inpatient and outpatient settings. The combination of these elements makes patients in critical care areas particularly vulnerable to medication errors and their potentially dire consequences.

\section{Medication errors in the ICU}

Medication errors in the ICU are more likely to lead to harm, and the severity of harm is greater in comparison to errors that occur outside of the ICU. ${ }^{16}$ Errors can occur at any stage of the medication use process, which is typically defined by discrete functions or nodes. These include prescribing, transcribing, dispensing, administering, and monitoring. Examining the points at which medication errors occur along the continuum of the medication use process allows for systematic analysis and identification of areas for improvement. ${ }^{20}$

A recently published analysis of voluntarily reported medication errors in ICU and non-ICU settings showed that medication errors occur most frequently in the administration phase. ${ }^{16}$ Administration is vulnerable to error because it is the last step in the process before the patient receives the medication. Errors associated with this node are least likely to be identified and intervened upon by other members of the health care team. ${ }^{16}$ Administration errors include errors associated with infusion rates, incorrect or omitted doses, administration time, and physiochemical incompatibility of parenterally administered products. ${ }^{20}$ Optimization of product design stands to decrease medication errors at multiple steps in the process but may be particularly helpful for mitigating errors that occur at the administration node.

\section{Challenges, problems, and errors related to product design}

Challenges related to drug formulation are specific to the various routes of medication administration. However, errors associated with medication appearance occur among all drug formulations and routes of administration.

\section{Medication labeling}

Medication vials, liquid medication cups, intravenous (IV) medication bags, and packaging that have similar labeling font, font size, and color scheme trigger misperception and mix-ups at every stage of the medication use process. These look-alike medications contribute to medication errors 
and are of utmost concern at the time of dispensing and administration. Containers within or across a product line should not be similar in appearance. Different strengths and product or vial sizes should be distinguishable by size, color, shape, or some other mechanism. ${ }^{23}$

Product labeling is an issue that spans across all routes of administration. Labeling includes package inserts, medication guides, product packaging, and marketing materials. The IOM identified a number of ways in which labeling can be problematic, including look-alike sound-alike drug names, different formulations of the same drug, multiple abbreviations for the same concept, confusing abbreviations and symbols, unclear dose strength designations, cluttered labeling, inadequate prominence of warnings, and lack of standardized terminology. ${ }^{6}$ These labeling issues have contributed to serious medication errors. ${ }^{6}$

Medication name nomenclature has been associated with dispensing errors. Some suggestions to reduce product namerelated errors have included modifying workflow to minimize distractions and applying technological solutions, such as font variation, use of tall man lettering, automated alerts, and barcode scanning. ${ }^{24}$ Lexical similarity is measurable, and predictive models exist to determine the risk of sound-alike medication names. Automated measures of similarity should be undertaken before medication marketing and approval to minimize this risk. ${ }^{25}$

The IOM has provided several recommendations to help address these problems. ${ }^{6}$ One recommendation was that FDA and the pharmaceutical industry develop a standardized drug nomenclature and apply failure modes and effects analysis (FMEA) to labeling. An FMEA is a prospective systematic quality improvement process by which a multidisciplinary team explores the use of new products. This would expose potential failures in labeling design and their relative impact. ${ }^{26}$ The IOM also recommended increasing efforts to develop and study optimum designs of drug labeling, evaluating the impact of free medication samples, and expanding the availability of unit-of-use packaging.

Some problems related to drug formulation design are specifically associated with a particular route of administration. These challenges, as well as some potential solutions, will be addressed separately based on route of administration.

\section{Route-specific problems related to drug formulation design}

\section{Enteral route of administration}

Access issues related to enterally administered medications are encountered frequently in critical care. Medications that are only available in an enteral formulation pose a problem for patients with a strict nothing by mouth (NPO) order. Carbidopa/levodopa, which is only available as an enteral formulation, is one example. A patient with Parkinson's disease who is admitted to the ICU and given a strict NPO order has no alternative way to receive this critical medication, and concessions have to be made to allow enteral administration of the drug.

Related to this challenge is the problem of medication administration via small-bore feeding tubes. Because of the small lumen, these tubes are prone to becoming clogged and, as many are placed in the duodenum or jejunum, they are also difficult to replace. ${ }^{27}$ For these reasons, clinicians often decide against administering crushed medications via small bore feeding tubes. Some medications are available as manufactured suspensions for enteral administration, but most are not. Pharmacies can prepare suspensions of some medications when they are not commercially available, but not all drugs can be adapted to a suspension, and stability information is often limited.

Another issue related to enteral access is immediaterelease (IR) versus extended-release (ER) formulations of medications. These different formulations are often confusing for clinicians, and the differences between the formulations vary from drug to drug. For a patient who receives medications via a nasogastric tube, it would be appropriate to crush a metoprolol tartrate tablet (the IR formulation), but crushing a metoprolol succinate tablet (the ER formulation) could lead to a more rapidly profound, yet unsustained, effect than is desired. The problem of tablet crushing is not unique to drugs with an IR and ER formulation. A number of medications should not be crushed for a variety of reasons, ranging from onset of effect to cytotoxic potential. ${ }^{28}$

In addition to tablet crushing, tablet splitting is a source of challenges and errors. Tablet splitting involves many of the same risks as tablet crushing, but it can also cause inaccurate dosing. One study that evaluated the practice of tablet splitting found a deviation of up to $58 \%$ from the intended medication dose when a commercially available tablet splitter was used. ${ }^{29}$

Absorption is another commonly cited issue with enteral administration of a medication in critical care. Medications administered via a nasojejunal or jejunostomy tube bypass the duodenum, the principle absorption site for most medications, leading to variable effects on absorption and first-pass metabolism. ${ }^{30}$ The absorption of some medications can be affected by concomitant administration with enteral nutrition (eg, warfarin, phenytoin, levothyroxine), and so special care 
must be taken to appropriately interrupt enteral nutrition around the timing of drug administration. ${ }^{31-33}$

Even if a medication is able to be administered to the stomach or duodenum, small bowel resection, gastroparesis, ileus, and decreased splanchnic flow all have the potential to affect absorption and efficacy. However, clinicians may also overestimate the absorptive problem and decline to use an enteral formulation in a patient who could actually benefit from it. Additional research into how these problems affect medication absorption in critically ill patients would help guide decision-making in this population.

In consideration of these issues, several qualities would be advisable for the ideal enterally administered medication. It would be available in enteral and parenteral formulation. It would be available as a suspension and as a tablet or capsule. If the drug had both IR and ER formulations, the difference would be clear and the labeling would adequately distinguish between the two.

\section{Subcutaneous route of administration}

Accuracy of administration, specifically the depth of injection, is a concern with medications administered subcutaneously. A health care provider may intend to give a subcutaneous injection, but actually inject much deeper into the muscle. Significant differences in absorption, onset of effect, and duration of action have been noted with insulin administered intramuscularly as compared to subcutaneously. ${ }^{34,35}$ These differences can lead to unnecessary complications in a patient's treatment.

Variable absorption is also a concern with subcutaneously-administered medications in critically ill patients. It has been suggested that hemodynamic instability, vasoactive medications, and fluid shifts may alter the absorption of medications such as subcutaneous insulin and heparin, but the data are conflicting. ${ }^{36-38}$ More information is needed to guide therapy with subcutaneously administered medications in this patient population.

The ideal subcutaneously administered medication would be distributed along with needles of the appropriate length for subcutaneous administration. It would also have labeling that clearly denotes that it is intended for subcutaneous administration. Additionally, the prescribing information would include details about pharmacokinetics and pharmacodynamics specifically in critically ill patients.

\section{Intramuscular route of administration}

Intramuscular medications are subject to the same problem of administration accuracy as subcutaneous medications.
Inadvertent administration of a these medications to tissue other than muscle could lead to differences in absorption and effect. Additionally, many critically ill patients experience muscle wasting, which further complicates this route of administration. ${ }^{39,40}$

Intramuscular administration of medications poses a risk of hematoma in any patient. ${ }^{41}$ In the critically ill population, where many patients are anticoagulated or coagulopathic, the concern for hematoma is even greater. Although data suggest intramuscular injection may be safe in anticoagulated patients, it is generally avoided whenever possible. ${ }^{42}$

The ideal intramuscularly administered medication would be distributed along with needles of the appropriate length and would also include labeling to indicate the appropriate muscular bed for administration.

\section{Intravenous route of administration}

Patients in the ICU are more likely than others to be prescribed IV medications. Thus IV medications commonly administered to critically ill patients should be a key target for optimal drug formulation design. ${ }^{20}$ Because medications delivered via the IV route pharmacokinetically bypass an absorption phase, they cause systemic effects within seconds of administration, making this route especially important for critically ill patients. These same characteristics also leave patients vulnerable to harm from IV-associated medication errors. Incorrect dosing and rates, including unintentional bolus administration, are common errors encountered with IV medication administration. ${ }^{43}$

IV medications that require further dilution before safe administration should never be packaged in a manner that suggests or could allow for direct administration (eg, prefilled syringes). Additionally, medications that require mixing before use or administration should be avoided, and when absolutely necessary, should be labeled as such. ${ }^{23}$ A number of medications in the ICU are provided via continuous infusion. As a result, large fluid volumes might be administered to patients who are already volume overloaded or have one or more electrolyte abnormality. The requirement for a carrier fluid may complicate this challenge even further in critically ill patients. The use of a high carrier rate allows the drug to reach the patient quickly and minimizes the time to a systemic effect after a change in rate, especially with vasoactive medications. However, use of a high carrier rate increases the risk of infusing an unintentional medication bolus when changes are made to either the carrier rate or drug infusion rate. ${ }^{44}$ Ideally the medications delivered to ICU patients should be easily titratable, meaning they have a rapid onset; 
have a short half-life; are supplied in standard-concentration, inexpensive, ready-to-use bags; and are not extremely concentrated (requiring a carrier fluid) or diluted (minimizing the volume required to deliver the drug).

Smart infusion pumps with integrated decision support represent a targeted approach to minimize IV-associated medication errors. However, the presence of smart infusion pumps alone does not decrease serious medication errors. Smart infusion pumps were designed to promote safety and simplify medication administration. These pumps can store large drug libraries with information about weight-based dosing, standard infusion rates, and maximum infusion rates. They can also enforce these parameters to prevent inappropriate infusion rates and errors. Some pumps can be accessed wirelessly, allowing an entire fleet of pumps to be updated simultaneously.

Smart infusion pumps have drawbacks as well. Many safety features can be bypassed by manual overrides. This is necessary in certain situations but can be overused. A catalog of standard concentrations should be used to eliminate the need to deviate from the drug library within smart infusion pumps. The extensive drug libraries require constant maintenance to keep up with ever changing formularies and policies. The process of updating a fleet can be difficult, even with a wirelessly accessible pump. If a pump is sitting in an alcove without access to a wireless signal, it will not be updated with the rest of the fleet. Some of these issues likely contribute to the lack of benefit seen in some studies that have compared smart pumps with standard infusion pumps. ${ }^{44}$

In 2013, the American Society of Health-Systems Pharmacists adopted a policy statement encouraging the development of "nationally standardized drug concentrations and dosing units for commonly used high-risk drugs that are given as continuous infusions to adult and pediatric patients ..."45

Smart infusion pump technology must be accompanied by a culture that utilizes the technology, making it difficult to bypass the preprogrammed drug library, and adequately supports the bedside nurse with policies and protocols that guide rates of administration and appropriate titration, thereby minimizing the effect of human performance. ${ }^{43}$

\section{Transdermal route of administration}

A number of safety concerns surround use of the transdermal route in critically ill patients. Transdermal drug delivery is erratic in critically ill patients. Because perfusion to epidermal and subcutaneous tissue is often lower than normal, it can cause unpredictable and often less-than-optimal absorption.
On the other hand, elevated core temperature and febrile states, which are not uncommon in critically ill patients, increase absorption and the risk of excessive drug release. Many patches include aluminum backing, making them unsafe for wear in a magnetic resonance imaging machine. It is for these reasons that medication delivery via the transdermal route should largely be avoided in critically ill patients. If patients are admitted to the ICU wearing a transdermal medication patch, it should be discontinued as soon as possible, and other more reliable routes should be utilized. ${ }^{46}$ The transdermal route should not be a target for novel drug delivery in the critically ill patient population.

\section{Epidural route of administration}

The American Society of Anesthesiologists practice guidelines for acute pain management in the perioperative setting recommend the use of a multimodal approach to pain management, including neuraxial opioids, systemic opioid patient controlled analgesia, and peripheral regional techniques. Evidence has shown that using a multimodal approach minimizes postoperative pain and systemic opioid requirement ${ }^{47}$ However, the guidelines state that use of these therapies should be accompanied by institutional policies and procedures that provide for education and training of health care providers and documentation that patient-specific and institutional outcomes are monitored. ${ }^{47}$

Local anesthetics (eg, bupivacaine, ropivacaine) must be used with caution in the ICU. Anesthesiologists must first verify that epidural catheters are indeed in the epidural space. Even when infused appropriately, local anesthetics administered via the epidural route can cause systemic vasodilation and hypotension. In critically ill patients, the risks and benefits should be weighed carefully. It may be most appropriate to use separate infusions for local anesthetics and systemic opioids because the clinician can titrate each individually to minimize adverse effects. However, inadvertent systemic administration of local anesthetics can cause significant cardiotoxicity that could lead to arrhythmias, disturbances in contractility, or even cardiac arrest. ${ }^{48}$ A number of safety measures must be in place to prevent such mistakes. If not available commercially, infusions should be compounded in the pharmacy and not in the operating room or ICU. Medication bags and syringes should be labeled clearly with information regarding the route of administration if other than IV (eg, wording such as "epidural use only"). Independent double-checks should be implemented at the bedside when epidural medications are initiated and when rates or doses are changed. 


\section{System-based improvements to mitigate errors}

In addition to the specific challenges associated with medication routes outlined previously, a multimodal approach is required to mitigate medication-related errors in the ICU. While not specific to medication route, they are equally important and include issues amenable to human factors engineering, such as unique ports for routes of administration, local technologies such as computerized provider order entry (CPOE), decision support systems, and barcode administration. System-level approaches such as utilization of telemedicine, simulation-based education, improvements in patient safety culture to optimize detection and reporting, and the use of FMEA and simulation-use testing are additional strategies aimed at decreasing medication errors.

\section{Unique ports for different routes of administration}

The Joint Commission, World Health Organization, and other organizations have released warnings and recommendations regarding the problem of medications being administered via incorrect routes. ${ }^{49,50}$ To address this problem, unique interlocking mechanisms are being developed to distinguish enteral, IV, and intrathecal routes. These ports prevent lines intended for two different routes from being connected (eg, connecting an enteral nutrition line to a central catheter). Specific labeling of ports is also recommended to prevent errors, as is the use of different dedicated infusion pumps. This new technology is being accepted widely and becoming a standard of care.

\section{Technology}

The last few decades have seen technology play an increasing role in health care. New tools are regularly introduced with the goal of mitigating harm. While well-intentioned, care must be taken when using a new tool as they can introduce new sources of error in existing processes by changing task performance or facilitating unfamiliar behaviors.

\section{Computerized provider order entry}

$\mathrm{CPOE}$, the process by which a health care provider enters an electronic order into a computer system, has dramatically influenced clinical practice and medication safety. Despite the advantages of CPOE, many challenges still remain. Prebuilt orders and order sets can be too rigid and prevent individualized care in unique situations. Additionally, initial implementation, transition between, or upgrading health care software packages pose patient safety risks. When vital information is unable to be located by frontline staff, or if information exists in only one of multiple software systems, patient histories may not be complete, and patient safety is at risk. While implementation of new CPOE systems comes with new challenges, many problems and errors stemming from orders written on paper are being eliminated. ${ }^{20} \mathrm{CPOE}$ systems can be developed to guide providers toward appropriate medication regimens. Prebuilt orders with common doses, frequencies, and routes of administration provide direction when initiating a regimen. Order sets can group orders together to streamline processes for frequently repeated tasks. CPOE systems that interface with the pharmacy order verification system can remove the problematic step of retranscription, allowing pharmacists and nurses to focus more on clinical responsibilities. All of these advancements can improve safety and efficiency.

\section{Decision support software}

The incorporation of decision support software into CPOE programs has added an extra layer of safety and efficiency to the order-writing process. Warnings about drug interactions, impaired renal clearance, standard dosing, and other issues can address potential errors and adverse events immediately. ${ }^{51}$ Pharmacists can receive these alerts when reviewing orders and proactively contact prescribers with recommendations. By giving the prescribers these alerts in real-time, the software has the potential to reduce the frequency of inappropriate orders and reduce the number of phone calls for clarification. However, "alert fatigue" is a concern with decision-support software. If the software is programmed to give an excessive number of warnings, prescribers are less likely to read and react to them. ${ }^{52}$ The warnings supplied by the system must be reviewed on an ongoing basis and appropriate adjustments made to reduce the number of unnecessary alerts.

\section{Barcode medication administration}

Barcode medication administration provides a double-check to verify the medication, dose, route, patient, and dosing time. These barcode systems can interface with the electronic medication administration record to automatically chart a dose when the barcode is scanned. These systems improve safety and efficiency simultaneously. ${ }^{53}$

However, not all medications will have barcodes, such as unit dose items made from a bulk container. Therefore, pharmacies must be able to produce barcodes that can interface with the system. Another problem is the issue of automatic charting; if a nurse scans a medication but does 
not immediately administer it to the patient, it will be charted as "given" when in actuality it might have been forgotten. Encouraging nurses to scan medications only immediately before administration would help to address this issue.

\section{Telemedicine}

The use of telemedicine has spread rapidly over the last decade, particularly in the ICU setting. ${ }^{54}$ It allows clinicians to assess and monitor patients using technology applications and to provide information and participate in multiple aspects of patient care. However, some uncertainty surrounds the specific ways that telemedicine in ICUs can directly benefit patient care.

Telemedicine can potentially minimize medication errors that occur when access to experienced, specialized staff is lacking. Studies show that tele-ICU clinicians improve medication management and safety. ${ }^{55}$ Physician-related medication errors were shown to be significantly lower in patients who received telemedicine consults rather than those who received telephone consults or no consultation at all. ${ }^{56}$ Reductions in reported medication errors were also noted when a clinical pharmacist was part of the tele-ICU team. ${ }^{57}$

Although telemedicine is promising, its specific role and impact, especially in the ICU, on medication management and rates of medication errors and ADEs needs to be quantified to justify the costs.

\section{Simulation for education}

While significant research exists on the causes of medication errors, there is a significant lack of research on staff reporting of medication errors and the steps taken to prevent future occurrence. ${ }^{58}$ Studies show that self-reporting of medical errors falls far short of direct observation. ${ }^{59,60}$ Educational strategies to address this ideally need to heighten error awareness, set expectations and goals, and provide appropriate feedback. ${ }^{60,61}$ Social, cognitive, and emotional capabilities that complement technical skills and comprise skills such as decision making, teamwork, situation awareness, and task management should be emphasized. ${ }^{3}$

Education of medical and nursing staff, particularly by clinical pharmacists, is a vital part of a strategy to prevent medication errors. ${ }^{62-64}$ Simulation-based education has been shown to be more interactive and pragmatic than traditional didactic sessions. Along with the assessment of technical skills, it allows the development of nontechnical skills. It facilitates the use of real-time assessment, practical and clinical judgment, as well as psychomotor and communication skills to optimize understanding of material and improve task execution. ${ }^{65-67}$ Such an approach has the potential to not only prevent similar errors from recurring but to improve health care provider awareness to detect medication errors in the first place.

A direct comparison between medication administration error rates in two ICUs before and after the provision of either didactic or simulation-based sessions showed a significant sustained reduction in medication error rates in the simulation-based group compared to the didactic group. ${ }^{68}$ This reduction occurred despite similar improvements in quiz scores of the two study arms, demonstrating that participants understood the content equally well. Another recent study used simulation to demonstrate mitigation of interruptionrelated medication administration errors. ${ }^{69}$ Such learning methodologies hold promise as a strategy to reduce medication errors in a variety of settings.

\section{Safety culture and reporting}

Substantial evidence supports the need for an organizational commitment to improve patient safety as a whole, including medication errors. ${ }^{6}$ Studies have found that the safety climate of a unit is predictive of medication error incidence; a more positive culture is associated with fewer errors. ${ }^{70,71}$ Proposed mechanisms for improving outcomes are varied. A robust culture of safety might improve adherence to best practices at the various stages of the medication process and encourage a positive and open response to errors. ${ }^{70}$ Fear of negative consequences can be a major barrier to accurate reporting of errors, with as many as $50 \%$ to $96 \%$ going unreported. ${ }^{72-74}$ Instead of improving patient safety, a punitive culture causes providers to hide their mistakes, thereby preventing recognition, evaluation, and improvement of underlying causes and system issues. ${ }^{75,76}$

A primary component of establishing an organizational culture of safety is moving away from a punitive or blame culture to a nonpunitive or just culture. A recent review noted that only half of existing medication error reporting systems were considered nonpunitive. This high rate of punitive systems is a major barrier to reporting medication errors and limits their effectiveness as tools for improvement and learning. ${ }^{77}$ To benefit fully from technologies that aid in preventing medication errors, a culture of safety is considered essential. ${ }^{78}$

\section{The role of formulation design}

Ideally, proactive steps to minimize risk should be undertaken during the drug product design process. Pharmaceutical companies should conduct systematic risk assessments that take 
into account human and environmental factors to identify opportunities for error prior to marketing and production. If any issues arise, pertinent design changes can be made. Two tools that may be helpful in this process include FMEA and simulated-use testing. ${ }^{23}$ FMEA facilitates a systematic approach to the identification and prioritization of all possible defects within the product design process. ${ }^{26}$ In simulated-use testing, end users carry out tasks in real-life scenarios with the labeled drug products. These scenarios include each node of the medication use process. Data are collected through direct observation and solicitation of subjective feedback from representative participants. ${ }^{23}$ The results of simulateduse testing should be integrated into analytic approaches such as FMEA to further elucidate use-related risks that could not have been predicted. ${ }^{23}$ To develop safe medications, companies must prospectively consider the end-user, and these tools offer a mechanism by which to do so.

\section{Conclusion}

Medication safety is a priority for clinicians who care for patients, especially those who are most critically ill. Clearly, many challenges exist in current drug formulation design, but optimal drug formulation can mitigate errors at multiple points of the medication use process. Risk factors that predispose end users to errors can be minimized or eliminated by optimizing drug formulations. This must become a priority for drug manufacturers.

However, any approach to address the burden of medication errors to improve patient safety needs to be comprehensive. Because it is a large, complex problem, no single recommendation can be considered a definitive solution. Equally as important as drug product design are system-based solutions and minimization of opportunities for errors using approaches based in methodologies such as human factors engineering. Systems must be developed and technology must be utilized to enable users to interact with and utilize products in a safe way. Leveraging technology must continue to be a priority among hospitals and health-systems, including the safe implementation of CPOE, clinical decision support, smart infusion pumps, and barcode medication administration. Prospective, proactive assessment must be undertaken to minimize the cost associated with redesigns and, most importantly, minimize potential harm to patients.

Ultimately, every ICU is unique and needs to explore its own medication errors, those actually reported as well as opportunities for error that might not have been reported. This will allow the approach to error mitigation to be tailored to the needs of the unit, its staff, and its patients. Multimodal approaches for improvement can then be developed, implemented, and reviewed to achieve optimal results.

\section{Acknowledgment}

The authors would like to thank Claire Levine, MS, ELS, from the Johns Hopkins Department of Anesthesiology and Critical Care Medicine for her tremendous assistance with the preparation of the manuscript.

\section{Disclosure}

The authors report no conflicts of interest in this work.

\section{References}

1. Committee on Quality of Health Care in America, Institute of Medicine. To err is human: building a safer health system [webpage on the Internet]. Washington, DC: National Academies Press; 2000. Available from: http://www.nap.edu/catalog.php?record_id=9728. Accessed February 28, 2014.

2. Centers for Disease Control and Prevention, National Center for Health Statistics. Births and deaths: preliminary data for 1998. National Vital Statistics Reports. 1999;47(25):6.

3. Brennan TA, Leape LL, Laird NM, et al. Incidence of adverse events and negligence in hospitalized patients. Results of the Harvard Medical Practice Study I. N Engl J Med. 1991;324(6):370-376.

4. Leape LL, Brennan TA, Laird N, et al. The nature of adverse events in hospitalized patients. Results of the Harvard Medical Practice Study II. N Engl J Med. 1991;324(6):377-384.

5. Phillips DP, Christenfeld N, Glynn LM. Increase in US medication-error deaths between 1983 and 1993. Lancet. 1998;351(9103):643-644.

6. Committee on Identifying and Preventing Medication Errors and the Board on Health Care Services. Aspden P, Wolcott JA, Bootman JL, Cronenwett LR, editors. Preventing Medication Errors. Quality Chasm Series; 2007.

7. Ernst FR, Grizzle AJ. Drug-related morbidity and mortality: updating the cost-of-illness model. J Am Pharm Assoc (Wash). 2001;41(2): 192-199.

8. Secretary's Advisory Committee on Regulatory Reform. Bringing Common Sense to Health Care Regulation: Report of the Secretary's Advisory Committee on Regulatory Reform; 2002. Washington, DC: Secretary's Advisory Committee on Regulatory Reform. Available from: http://www.rwhc.com/papers/DHHS.RRAC.11.21.02.Report.pdf. Accessed February 28, 2014.

9. Cullen DJ, Sweitzer BJ, Bates DW, Burdick E, Edmondson A, Leape LL. Preventable adverse drug events in hospitalized patients: a comparative study of intensive care and general care units. Crit Care Med. 1997;25(8):1289-1297.

10. Lester H, Tritter JQ. Medical error: a discussion of the medical construction of error and suggestions for reforms of medical education to decrease error. Med Educ. 2001;35(9):855-861.

11. Rothschild JM, Landrigan CP, Cronin JW, et al. The Critical Care Safety Study: The incidence and nature of adverse events and serious medical errors in intensive care. Crit Care Med. 2005;33(8):1694-1700.

12. Abramson NS, Wald KS, Grenvik AN, Robinson D, Snyder JV. Adverse occurrences in intensive care units. JAMA. 1980;244(14): 1582-1584.

13. Ridley SA, Booth SA, Thompson CM; Intensive Care Society's Working Group on Adverse Incidents. Prescription errors in UK critical care units. Anaesthesia. 2004;59(12):1193-1200.

14. Herout PM, Erstad BL. Medication errors involving continuously infused medications in a surgical intensive care unit. Crit Care Med. 2004;32(2):428-432. 
15. Garrouste-Orgeas M, Philippart F, Bruel C, Max A, Lau N, Misset B. Overview of medical errors and adverse events. Ann Intensive Care. 2012;2(1):2.

16. Latif A, Rawat N, Pustavoitau A, Pronovost PJ, Pham JC. National study on the distribution, causes, and consequences of voluntarily reported medication errors between the ICU and non-ICU settings. Crit Care Med. 2013;41(2):389-398.

17. Mehrotra R, De Gaudio R, Palazzo M. Antibiotic pharmacokinetic and pharmacodynamic considerations in critical illness. Intensive Care Med. 2004;30(12):2145-2156.

18. Gonçalves-Pereira J, Póvoa P. Antibiotics in critically ill patients: a systematic review of the pharmacokinetics of $\beta$-lactams. Crit Care. 2011;15(5):R206.

19. Hussain E, Kao E. Medication safety and transfusion errors in the ICU and beyond. Crit Care Clin. 2005;21(1):91-110, ix.

20. Kane-Gill S, Weber RJ. Principles and practices of medication safety in the ICU. Crit Care Clin. 2006;22(2):273-290, vi.

21. Pronovost P, Weast B, Schwarz M, et al. Medication reconciliation: a practical tool to reduce the risk of medication errors. J Crit Care. 2003;18(4):201-205.

22. Summa-Sorgini C, Fernandes V, Lubchansky S, et al. Errors Associated with IV Infusions in Critical Care. Can J Hosp Pharm. 2012;65(1): 19-26.

23. US Department of Health and Human Services, Food and Drug Administration, Center for Drug Evaluation and Research. Guidance for Industry: Safety Consideration for Product Design to Minimize Medication Errors. Draft Guidance; 2012. Silver Springs, MD: US Food and Drug Administration. Available from: http:/www.fda.gov/ downloads/Drugs/GuidanceComplianceRegulatoryInformation/ Guidances/UCM331810.pdf. Accessed February 28, 2014.

24. Emmerton LM, Rizk MF. Look-alike and sound-alike medicines: risks and 'solutions'. Int J Clin Pharm. 2012;34(1):4-8.

25. Lambert BL. Predicting look-alike and sound-alike medication errors. Am J Health Syst Pharm. 1997;54(10):1161-1171.

26. Institute for Safe Medication Practices. Failure mode and effects analysis (FMEA): a tool to help guide error prevention efforts [webpage on the Internet]. ISMP Medication Safety Alert ${ }^{\mathbb{\circledR}} ; 2001 ; 21(6)$. Available from: https://www.ismp.org/tools/FMEA.asp. Accessed February 28, 2014.

27. Scanlan M, Frisch S. Nasoduodenal feeding tubes: prevention of occlusion. J Neurosci Nurs. 1992;24(5):256-259.

28. Salmon D, Pont E, Chevallard H, et al. Pharmaceutical and safety considerations of tablet crushing in patients undergoing enteral intubation. Int J Pharm. 2013;443(1-2):146-153.

29. Verrue C, Mehuys E, Boussery K, Remon JP, Petrovic M. Tablet-splitting: a common yet not so innocent practice. J Adv Nurs. 2011;67(1):26-32.

30. Williams NT. Medication administration through enteral feeding tubes. Am J Health Syst Pharm. 2008;65(24):2347-2357.

31. Dickerson RN, Garmon WM, Kuhl DA, Minard G, Brown RO. Vitamin $\mathrm{K}$-independent warfarin resistance after concurrent administration of warfarin and continuous enteral nutrition. Pharmacotherapy. 2008;28(3):308-313.

32. Dickerson RN, Maish GO 3rd, Minard G, Brown RO. Clinical relevancy of the levothyroxine-continuous enteral nutrition interaction. Nutr Clin Pract. 2010;25(6):646-652.

33. Wohlt PD, Zheng L, Gunderson S, Balzar SA, Johnson BD, Fish JT. Recommendations for the use of medications with continuous enteral nutrition. Am J Health Syst Pharm. 2009;66(16):1458-1467.

34. Vaag A, Handberg A, Lauritzen M, Henriksen JE, Pedersen KD, BeckNielsen H. Variation in absorption of NPH insulin due to intramuscular injection. Diabetes Care. 1990;13(1):74-76.

35. Frid A, Gunnarsson R, Güntner P, Linde B. Effects of accidental intramuscular injection on insulin absorption in IDDM. Diabetes Care. 1988;11(1):41-45

36. Dörffler-Melly J, de Jonge E, Pont AC, et al. Bioavailability of subcutaneous low-molecular-weight heparin to patients on vasopressors. Lancet. 2002;359(9309):849-850.
37. Rommers MK, Van der Lely N, Egberts TC, van den Bemt PM. Anti-Xa activity after subcutaneous administration of dalteparin in ICU patients with and without subcutaneous oedema: a pilot study. Crit Care. 2006;10(3):R93

38. Cumbo-Nacheli G, Samavati L, Guzman JA. Bioavailability of fondaparinux to critically ill patients. J Crit Care. 2011;26(4): 342-346.

39. Puthucheary ZA, Rawal J, McPhail M, et al. Acute skeletal muscle wasting in critical illness. JAMA. 2013;310(15):1591-1600.

40. Latifi R. Nutritional therapy in critically ill and injured patients. Surg Clin North Am. 2011;91(3):579-593.

41. Greenblatt DJ, Allen MD. Intramuscular injection-site complications. JAMA. 1978;240(6):542-544.

42. Raj G, Kumar R, McKinney WP. Safety of intramuscular influenza immunization among patients receiving long-term warfarin anticoagulation therapy. Arch Intern Med. 1995;155(14):1529-1531.

43. Rothschild JM, Keohane CA, Cook EF, et al. A controlled trial of smart infusion pumps to improve medication safety in critically ill patients. Crit Care Med. 2005;33(3):533-540.

44. Lovich MA, Kinnealley ME, Sims NM, Peterfreund RA. The delivery of drugs to patients by continuous intravenous infusion: modeling predicts potential dose fluctuations depending on flow rates and infusion system dead volume. Anesth Analg. 2006;102(4):1147-1153.

45. American Society of Health-System Pharmacists ${ }^{\circledR}$. Standardization of Intravenous Drug Concentrations. In: ASHP Policy Positions 1982-2013. Policy 1306. Hawkins, Bruce, editor. 2013. Available from: http://www. ashp.org/DocLibrary/BestPractices/policypositionsonly2013.aspx. Accessed February 28, 2014.

46. Ball AM, Bellamy CJ. Pharmacokinetic alterations in the critically ill. In: Lanken PN, Manaker S, Kohl BA, Hanson CW 3rd, editors. The Intensive Care Unit Manual. 2nd ed. Philadelphia, PA: ElsevierSaunders; 2014.

47. American Society of Anesthesiologists Task Force on Acute Pain Management. Practice guidelines for acute pain management in the perioperative setting: an updated report by the American Society of Anesthesiologists Task Force on Acute Pain Management. Anesthesiology. 2012;116(2):248-273.

48. Grissinger M. Reducing the risk of deadly mixups with epidural and intravenous drugs. P T. 2012;37(8):432-434.

49. Joint Commission on Accreditation of Healthcare Organizations, USA. Tubing misconnections - a persistent and potentially deadly occurrence. Sentinel Event Alert. 2006;36:1-3.

50. World Health Organization. Avoiding Catheter and Tubing Mis-Connections. Patient Safety Solutions; 2007. Geneva: World Health Organization. Available from: http://www.who.int/patientsafety/solutions/ patientsafety/PS-Solution7.pdf. Accessed February 28, 2014.

51. Lainer M, Mann E, Sönnichsen A. Information technology interventions to improve medication safety in primary care: a systematic review. Int J Qual Health Care. 2013;25(5):590-598.

52. Cash JJ. Alert fatigue. Am J Health Syst Pharm. 2009;66(23): 2098-2101.

53. Leung AA, Denham CR, Gandhi TK, et al. A safe practice standard for barcode technology. J Patient Saf. Epub March 10, 2014.

54. Lilly CM, Thomas EJ. Tele-ICU: experience to date. J Intensive Care Med. 2010;25(1):16-22.

55. Khunlertkit A, Carayon P. Contributions of tele-intensive care unit (Tele-ICU) technology to quality of care and patient safety. J Crit Care. 2013;28(3):315.e1-315.e12.

56. Dharmar M, Kuppermann N, Romano PS, et al. Telemedicine consultations and medication errors in rural emergency departments. Pediatrics. 2013;132(6):1090-1097.

57. Meidl TM, Woller TW, Iglar AM, Brierton DG. Implementation of pharmacy services in a telemedicine intensive care unit. Am J Health Syst Pharm. 2008;65(15):1464-1469.

58. Boyle TA, Mahaffey T, Mackinnon NJ, Deal H, Hallstrom LK, Morgan H. Determinants of medication incident reporting, recovery, and learning in community pharmacies: a conceptual model. Res Social Adm Pharm. 2011;7(1):93-107. 
59. Shannon RC, DeMuth JE. Comparison of medication error detection methods in the long term care facility. Consult Pharm. 1987;2:148-151.

60. Varadarajan R, Barker KN, Flynn EA, Thomas RE. Comparison of two error-detection methods in a mail service pharmacy serving health facilities. J Am Pharm Assoc (2003). 2008;48(3):371-378.

61. Wiederholt JB, Schommer JC, Mount JK, McGregor TD, Braatz PD. The Wisonsin Pharmacy Self-Inspection Project: an application of Berwick's theory of continuous improvement. Am J Pharm Educ. 2002;66:27-36.

62. Horn E, Jacobi J. The critical care clinical pharmacist: evolution of an essential team member. Crit Care Med. 2006;34(Suppl 3):S46-S51.

63. Kane SL, Weber RJ, Dasta JF. The impact of critical care pharmacists on enhancing patient outcomes. Intensive Care Med. 2003;29(5): 691-698.

64. LeBlanc JM, Seoane-Vazquez EC, Arbo TC, Dasta JF. International critical care hospital pharmacist activities. Intensive Care Med. 2008;34(3):538-542.

65. Seybert AL, Barton CM. Simulation-based learning to teach blood pressure assessment to doctor of pharmacy students. Am J Pharm Educ. 2007;71(3):48.

66. Steadman RH, Coates WC, Huang YM, et al. Simulation-based training is superior to problem-based learning for the acquisition of critical assessment and management skills. Crit Care Med. 2006;34(1):151-157.

67. Tuttle RP, Cohen MH, Augustine AJ, et al. Utilizing simulation technology for competency skills assessment and a comparison of traditional methods of training to simulation-based training. Respir Care. 2007;52(3):263-270.

68. Ford DG, Seybert AL, Smithburger PL, Kobulinsky LR, Samosky JT, Kane-Gill SL. Impact of simulation-based learning on medication error rates in critically ill patients. Intensive Care Med. 2010;36(9): 1526-1531.
69. Prakash V, Koczmara C, Savage P, et al. Mitigating errors caused by interruptions during medication verification and administration: interventions in a simulated ambulatory chemotherapy setting. BMJ Qual Saf. Epub June 6, 2014.

70. Hofmann DA, Mark B. An investigation of the relationship between safety climate and medication errors as well as other nurse and patient outcomes. Personnel Psychol. 2006;59(4):847-869.

71. Naveh E, Katz-Navon T, Stern Z. Readiness to report medical treatment errors: the effects of safety procedures, safety information, and priority of safety. Med Care. 2006;44(2):117-123.

72. Potylycki MJ, Kimmel SR, Ritter M, et al. Nonpunitive medication error reporting: 3-year findings from one hospital's Primum Non Nocere initiative. J Nurs Adm. 2006;36(7-8):370-376.

73. Sexton JB, Thomas EJ, Helmreich RL. Error, stress, and teamwork in medicine and aviation: cross sectional surveys. BMJ. 2000;320(7237): 745-749.

74. Uribe CL, Schweikhart SB, Pathak DS, Dow M, Marsh GB. Perceived barriers to medical-error reporting: an exploratory investigation. J Healthc Manag. 2002;47(4):263-279.

75. Wolf ZR, Serembus JF. Medication errors: ending the blame-game. Nurs Manage. 2004;35(8):41-42, 44, 47.

76. Leape LL, Woods DD, Hatlie MJ, Kizer KW, Schroeder SA, Lundberg GD. Promoting patient safety by preventing medical error. JAMA. 1998;280(16):1444-1447.

77. Holmström AR, Airaksinen M, Weiss M, Wuliji T, Chan XH, Laaksonen R. National and local medication error reporting systems: a survey of practices in 16 countries. J Patient Saf. 2012;8(4):165-176.

78. National Research Council. Patient safety: achieving a new standard for care [webpage on the Internet]. Washington, DC: The National Academies Press; 2004. Available from: http://www.nap.edu/catalog. php?record_id=10863. Accessed February 28, 2014.
Clinical Pharmacology: Advances and Applications

\section{Publish your work in this journal}

Clinical Pharmacology: Advances and Applications is an international, peer-reviewed, open access journal publishing original research, reports, reviews and commentaries on all areas of drug experience in humans. The manuscript management system is completely online and includes a very quick and fair peer-review system, which is all easy to use.

\section{Dovepress}

Visit http://www.dovepress.com/testimonials.php to read real quotes from published authors. 